subsets were defined by flow cytometry. Healthy donors and patients with rheumatoid arthritis served as controls.

Results: We observed that ATA- and ACA-positive SSc patients harbour circulating B cells that secrete either ATA-IgG or ACA-IgG upon stimulation, depending on their serotype. In addition, we noted spontaneous secretion of ATA-IgG and, more remarkably, extensive secretion of ATA-IgA in ATA-positive patients. This degree of spontaneous, antigen-specific IgA secretion was specific for the ATA response in ATA-positive patients, while spontaneous ACA-IgA secretion was undetectable in the ACA-positive patient group. FACS experiments showed that spontaneously ATA-IgA secreting B cells were primarily present in the plasmablast compartment.

Conclusion: Our findings demonstrate that ATA-positive SSc patients harbour an activated ATA-IgG and ATA-IgA B cell response, as indicated by the spontaneous secretion of both ATA isotypes by circulating plasmablasts. Remarkably, the ACA B cell response was far less active and lacked the active IgA component which suggests a difference in the triggers driving these autoreactive $B$ cell responses in patients. Moreover, the remarkable ATA-IgA secretion points towards a potential mucosal origin of the ATA response.

Disclosure of Interests: Corrie Wortel: None declared, Nina van Leeuwen: None declared, Maaike Boonstra: None declared, Rene Toes: None declared, Thomas Huizinga Grant/research support from: Ablynx, Bristol-Myers Squibb, Roche, Sanofi, Consultant of: Ablynx, Bristol-Myers Squibb, Roche, Sanofi, Jeska de Vries-Bouwstra: None declared, Hans Ulrich Scherer Grant/research support from: Bristol Myers Squibb, Sanofi, Pfizer, Speakers bureau: Pfizer, Lilly, Roche, Abbvie

DOI: 10.1136/annrheumdis-2020-eular.6049

\section{SAT0298 IS INTERLEUKIN 6 A FACTOR OF FIBROGENESIS IN DERMAL FIBROBLASTS?}

A. S. Siebuhr ${ }^{1}$, P. Juhl ${ }^{1}$, M. Karsdal ${ }^{2}$, A. C. Bay-Jensen ${ }^{1} .{ }^{1}$ Nordic Bioscience, ImmunoScience, Herlev, Denmark; ${ }^{2}$ Nordic Bioscience, R\&D, Herlev, Denmark

Background: Interleukin 6 (IL-6) is known to have both pro- and anti-inflammatory properties, depending on the receptor activation. The classical IL-6 signaling via the membrane bound receptor is mainly anti-inflammatory, whereas signaling through the soluble receptor (sIL-6R) is pro-inflammatory/pro-fibrotic. However, the direct fibrotic effect of IL-6 stimulation on dermal fibroblasts is unknown.

Objectives: We investigated the fibrotic effect of IL-6 + sIL-6R in a dermal fibroblast model and assessed fibrosis by neo-epitope biomarkers of extracellular matrix proteins.

Methods: Primary healthy human dermal fibroblasts were grown for up to 17 days in DMEM medium with $0.4 \%$ fetal calf serum, ficoll (to produce a crowded environment) and ascorbic acid. IL-6 [1-90 nM]+SIL-6R [0.1-9 nM] alone or in combination with TGF $\beta[1 \mathrm{nM}$ ] were tested in three different donors. TGF $\beta$ [1 nM], PDGF-AB [3 nM] and non-stimulated cells (w/o) were used as controls. Tocilizumab (TCZ) with TGF $\beta$ + IL-6 + sIL-6R stimulation was tested in one donor. Collagen type I, $\mathrm{III}$ and VI formation (PRO-C1, PRO-C3 and PRO-C6) and fibronectin (FBN-C) were evaluated by validated ELISAs (Nordic Bioscience). Western blot analysis investigated signal cascades. Gene expression of selected ECM proteins was analyzed. Statistical analyses included One-way and 2-way ANOVA and area under the curve analysis.

Results: formation by the end of the culture period. The fibronectin and collagen type VI signal were consistent between the three tested donors, whereas the formation of type III collagen was only increased in one donor, but in several trials. Type I collagen formation was unchanged by IL- 6 + sIL-6R stimulation. The gene expression of type I collagen was induced by IL-6 + sIL-6R. Western blot analysis validated trans-signaling by the IL-6+sIL-6R stimulation as expected.

IL-6 + sIL-6R stimulation in combination with TGF $\beta$ decreased fibronectin levels compared to TGF $\beta$ alone but did not reach the level of unstimulated fibroblasts. The formation of collagen type IV was generally unchanged with IL-6 + sIL-6R + TGF $\beta$ compared to TGF $\beta$ alone. Collagen type I and III formation was more scattered in the signals when IL- 6 + SIL-6R was in combination with TGF $\beta$, as the biomarker level could be either decreased or increased compared to TGF $\beta$ alone. In two studies the type I collagen level was synergistic increased by IL- 6 + sIL-6R + TGF $\beta$, whereas another study found the level to be decreased compared to TGF $\beta$ alone. The gene expression of fibronectin and type I collagen was increased with TGF $\beta+$ IL-6+sIL-6R compared to TGF $\beta$ alone.

Inhibition of IL-6R by TCZ in combination with IL-6 + sIL-6R did only decrease the fibronectin level with the lowest TCZ concentration $(p=0.03)$. TCZ alone decreased the fibronectin level in a dose-dependent manner (One-way ANOVA $\mathrm{p}=0.0002$ )

Conclusion: We investigated the fibrotic response of dermal fibroblasts to IL-6 + sIL-6R stimulation. IL-6 modulated the fibronectin level and modulated the collagen type III formation level in a somewhat dose-dependent manner. In combination with TGF $\beta$, IL- 6 decreased collagen type I and IV formation and fibronectin. However, in this study inhibition of IL-6R by TCZ did not change the fibrotic response of the dermal fibroblasts. This study indicated that IL-6 did not induce collagen formation in dermal fibroblasts, except type III collagen formation with high IL-6 concentration.

\section{Figure:}

Flbronectin remodeling by IL-6

in TGF $\beta$ stimulated dermal fibroblasts

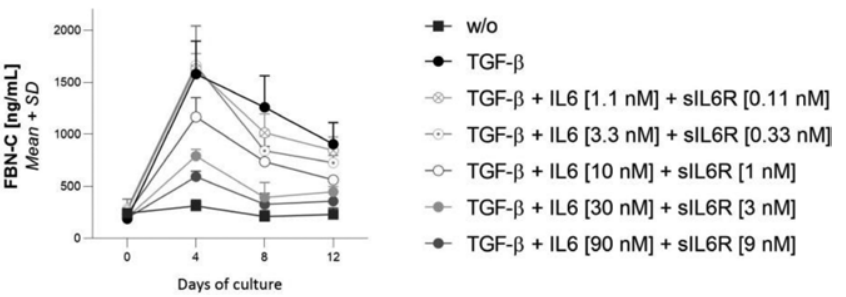

Gene expression in SiaJ

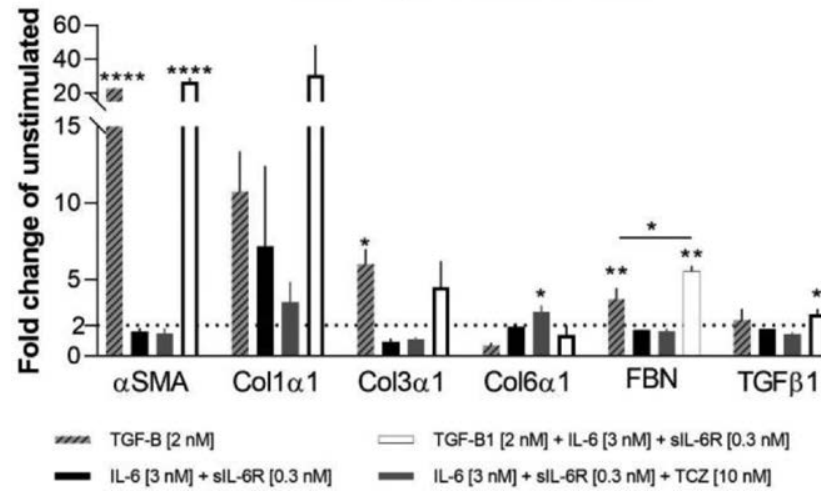

Disclosure of Interests: Anne Sofie Siebuhr Employee of: Nordic Bioscience, Pernille Juhl Employee of: Nordic Bioscience, Morten Karsdal Shareholde of: Nordic Bioscience A/S., Employee of: Full time employee at Nordic Bioscience A/S., Anne-Christine Bay-Jensen Shareholder of: Nordic Bioscience A/S Employee of: Full time employee at Nordic Bioscience A/S.

DOI: 10.1136/annrheumdis-2020-eular.4061

\section{SAT0299 PROLIFERATION, MIGRATION AND CONTRACTION ARE DIFFERENT BETWEEN TGFB AND PDGF STIMULATED DERMAL FIBROBLASTS}

A. S. Siebuhr ${ }^{1}$, S. F. Madsen ${ }^{1}$, M. Karsdal ${ }^{2}$, A. C. Bay-Jensen ${ }^{1}$, P. Juhl' ${ }^{1}{ }^{1}$ Nordic Bioscience, ImmunoScience, Herlev, Denmark; ${ }^{2}$ Nordic Bioscience, R\&D, Herlev, Denmark

Background: Systemic sclerosis has vascular, inflammatory and fibrotic components, which may be associated with different growth factors and cytokines. Platelet derived growth factor (PDGF) is associated with the vasculature whereas tumor necrosis factor beta (TGF $\beta$ ) is associated with inflammation and fibrosis. We have developed a fibroblast model system of dermal fibrosis for anti-fibrotic drugs testing, but the effect of the fibroblasts mechanistic properties are unknown.

Objectives: We investigated different mechanical capacities of PDGF and TGF $\beta$ treated human healthy dermal fibroblasts in the SiaJ setting

Methods: Primary human healthy dermal fibroblasts were grown in DMEM medium containing $0.4 \%$ fetal calf serum, ficoll (to produce a crowded environment) and ascorbic acid for up to 17 days. A wound was induced by scratching the cells at $0,1,3$ or 7 days after treatment initiation. Wound closure was followed for 3 days. Contraction capacity was tested by creating gels of human fibroblasts produced collagens containing dermal fibroblasts and contraction was assessed at day 2 by calculating the percentage of gel size to total well size. Collagen type I, III and VI formation (PRO-C1, PRO-C3 and PRO-C6) and fibronectin (FBN-C) 